\title{
Effective Recycling of Spent Auto Catalytic Converters by Using Electrochlorination Method
}

\author{
Nikolay Samotaev ${ }^{1, a}$, Andrey Antonov ${ }^{1}$, Grigory Tsarev ${ }^{1}$ and Andreas Tietz ${ }^{2}$ \\ ${ }^{1}$ National Research Nuclear University MEPhl (Moscow Engineering Physics Institute), 115409, Kasirskoe highway 31, Moscow, Russia \\ ${ }^{2}$ ReMetall Deutschland AG, 01994, Hauptstrasse 2a, Drochow, Germany
}

\begin{abstract}
Modern methods of recycling spent automotive catalysts and their main disadvantages in industrial practice are considered. The electrochlorination method is proposed as the basis of the platinum-group metals (PGM) recycling technology. As the test of proposed technology a few tons of spent automotive catalysts were processed. The results of the work on the extraction of platinum, palladium, rhodium are analyzed. The extraction rates during experiments were reached for $\mathrm{Pt}-97 \%, \mathrm{Pd}-97 \%$ and $\mathrm{Rh}-80 \%$.
\end{abstract}

\section{Introduction}

The main raw material source of PGM scrap is spent automobile catalysts. The annual consumption of PGM used in the production of automotive catalysts is: for Platinum (Pt) around 50 tons, for Palladium (Pd) 120 tons and for Rhodium (Rh) 15 tons. The percentage of using platinum group metals (PGM) in automotive catalysts from the total annual volume produced is: for $\mathrm{Pt}-30 \%$, $\mathrm{Pd}-60 \%$ and $\mathrm{Rh}-85 \%$.

At present, spent automobile catalysts is accumulating in the companies that are processing black and colour scrap and participating in the car recycling program. Collectors of this type of raw materials are interested in creating an independent production of the processing of spent automobile catalysts, which significantly increases the profitability of their business.

Hydrometallurgical methods with various author's chemical-technological variations, including protected by patents, have a standard set of shortcomings. First, it is a multi-stage process - activation of PGM by thermal stabilization (Fig.1) of the ceramic carrier $\left(\gamma-\mathrm{Al}_{2} \mathrm{O}_{3}\right.$ in $\alpha$ $\mathrm{Al}_{2} \mathrm{O}_{3}$ ), acid leaching or hydrochlorination of PGM, filtration and washing of pulp, precipitation of the concentrate from the solutions obtained, again filtration of the finished concentrate and the like.

Each operation takes place in a separate installation, which requires multiple overloads of raw materials. In addition, the acceptable recovery of PGM is achieved by circulating the acid or mixing the ceramic carrier. This, under conditions of the fineness of the raw state of raw materials, considerably complicates the hardware design process. Environmental problems are significant. In the process, large quantities of waste solutions are generated that require disposal. As a result, such a technological "luxury" can be afforded by large nobel metal refineries, but not by small enterprises that are raw material collectors.

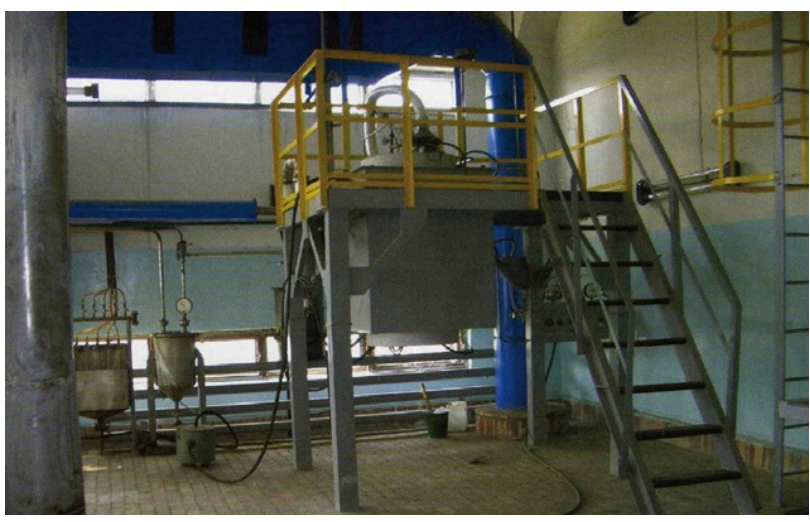

Figure 1. Photo of the furnace for activation of PGM by thermal stabilization of spent catalyst carrier.

Increasingly, in the choice of technological methods for processing catalysts in world practice gives preference to a two-stage scheme. The first stage is metallurgical (induction, arc, plasma) melting of a ceramic material with the addition of fluxes to a metalcollector. In this case, the PGM dissolve in the collector and in the form of metal of the ingot comes out of the process with an extraction of no higher than $97 \%$, but such a final extraction is achieved through a separate, and sometimes repeated, melting of the associated slag to isolate the residual PGM in collector. In addition, sometimes melting part of the PGM "hangs" in the dust filter and directly into the lining of the furnace, which requires their separate accounting and extractiontion. Thus, the direct output of PGM to the collector reaches, at best, $90-92 \%$. The main material of the collector is iron (or copper), which has a high solubility of PGM. The

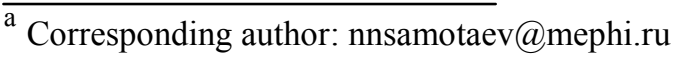


content of PGM in iron concentrate does not exceed $10 \%$. The low enrichment of this method neutralizes its main advantage, namely productivity, and certainly implies the presence of a stage of subsequent enrichment, which is a limiting scheme for this scheme. Besides, moreover, the energy costs of this process are significant $(2.5-3 \mathrm{kWh} /$ $\mathrm{kg}$ for raw materials, without taking into account the melting of slag), the hardware design is expensive.

At the second stage, the iron collector is refined (concentrate), where traditional hydrometallurgical schemes are used, where accompanied by the formation of a large number of liquid waste solutions. The extraction in this case is $98 \%$. Thus, the final the extraction of PGM from the spent automobile catalysts does not exceed $95 \%(\mathrm{Rh} \approx 85 \%)$, which is a low indicator. Each stage is performed, as a rule, at different enterprises and requires from the supplier of is spent automobile catalysts temporary and material costs, as well as the procedure for sampling and matching input analysis.

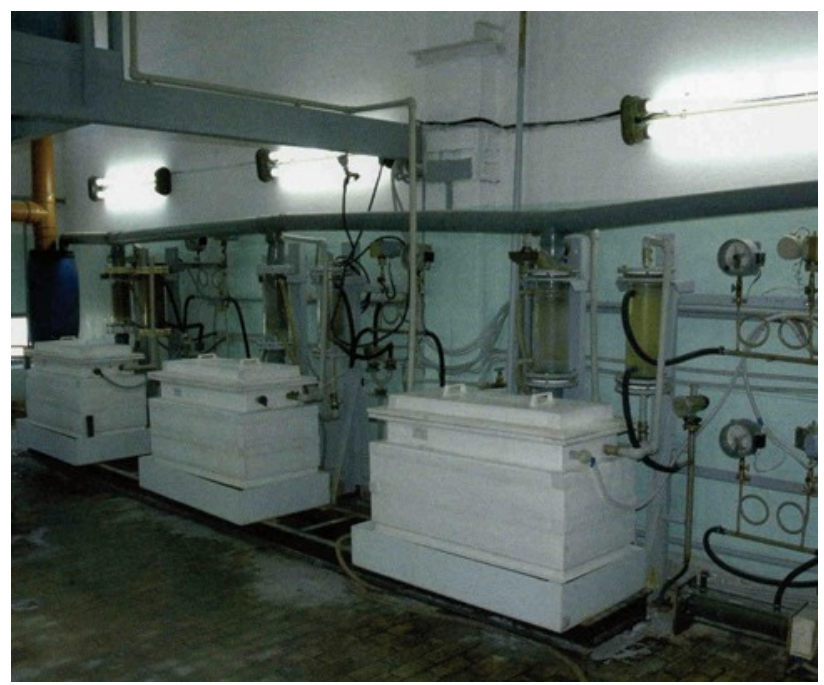

Figure 2. Photo of first generation of the horizontal electrochemical modules for recycling PGM.

\section{Experiments}

Our team solved the problem of developing in principle new technology, which has no analogues in the world industrial practice recycling of spent automobile catalysts containing PGM and combining the following advantages:

- Conducting a full cycle of processing in one hardware module;

- Achievement of direct output of PGM Pt - 98\%, Pd $98 \%$ and $\mathrm{Rh}-95 \%$ in concentrate not lower than $40 \%$ by amount of PGM;

- High productivity of one device (400 kg / per day) at low energy costs (1-1.5 kWh / kg);

- Ecological compatibility due to recycling of circulating fluids and effective utilization of gas emissions;

- Use of solid ceramic waste in the production of new products;

- The possibility of increasing productivity through the installation of additional processing modules.
Achieving such parameters makes this technology breakthrough in the secondary PGM industry. The method is based on the method of electrochlorination [1], which in industrial processes of recovery of secondary PGM (0.05- $0.2 \%$ by weight) has not been applied and has limited use in certain processes of gold recovery from pulps of mineral raw materials [2], affinity of rich concentrates PGM [3].

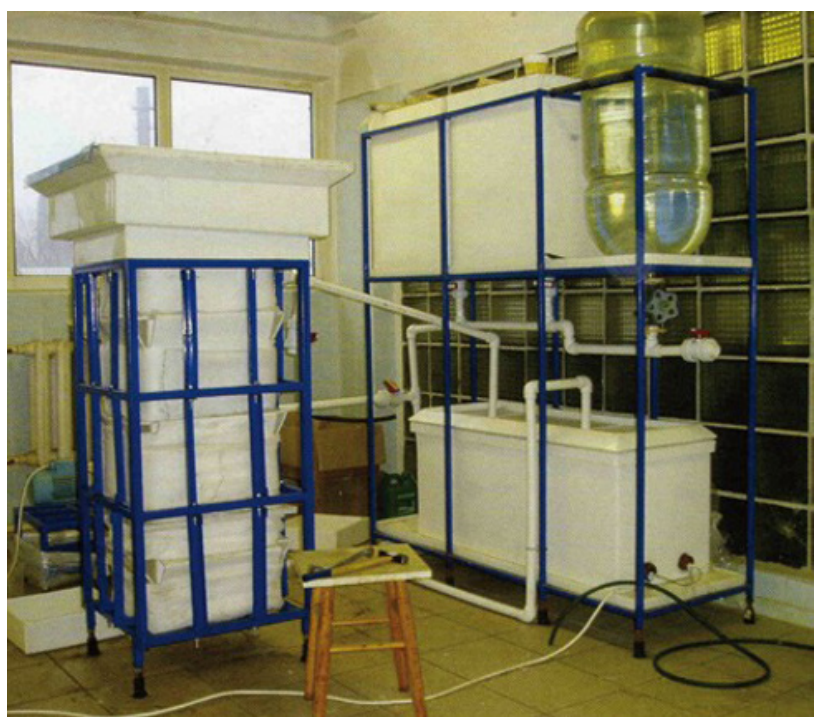

Figure 3. Photo of first generation of the vertical electrochemical module for recycling PGM.

During the development of the technology and the search for an optimal solution, the installations of the first generation [4,5] have been created, 10 tons of catalysts (mainly chemical and petrochemical catalysts). Extraction rates for $\mathrm{Pt}$ and $\mathrm{Pd}$ were achieved for spent catalysts $97 \%$, however for $\mathrm{Rh}$, not more than $80 \%$. Control of the residual content of PGM on a carrier was carried out at the ReMetall AG (Germany) using X-ray photoelectron spectroscopy and inductively coupled plasma atomic emission spectrometry. The analytical laboratory of the company conducted about 600 studies.

For depassivation of PGM in spent and grind catalysts carrier, (bulk density of raw materials of the order of 0,8 $\mathrm{kg} / 1$.) carried out their thermal activation in a specially designed furnace with an argon-hydrogen gas mixture temperature $700{ }^{\circ} \mathrm{C}$ for $2-4$ hours. Fig. 1 shows the general view of the furnace. After activation, the catalyst was pour forth into the interelectrode space of the electrolyser.

The hardware design at this stage of development had primitive constructive solution, the purpose of which was to confirm the effectives of the classical method of electrochlorination for raw material processing containing PGM. Figure 2 and 3 shows the horizontal and vertical installation of the first generation. The electrolyte installation of the first generation installations is water a solution of hydrochloric acid, which circulated in a closed loop (anode-cathode). Decomposition on the anode of hydrochloric acid due to the transmission of a constant current leads to the formation of reactive Chlorine, which enters in reaction with PGM, transferring 
them into a water-soluble form. As a result extraction of PGM into the electrolyte proceeds with their subsequent electrodepositing on the cathode. The resulting cathode deposit (concentrate based on the sum of PGM 40-70\%) was readily affinity in the laboratory. However, in addition to low extraction of $\mathrm{Rh}$, the technology and installations of the first generation had significant shortcomings, which limited the industrial introduction of the process in mass production.

\section{Discussions}

The limiting stage of the electrochemical process, which determines its productivity and acceptable values of extraction of $\mathrm{Pt}, \mathrm{Pd}$ and $\mathrm{Rh}$, is a long-term the electrodeposition PGM from a depleted solution, the duration of which was 24-50 hours. Circulation of electrolyte through finely dispersed raw materials constantly led to blockages of the separation membrane, which acts as a filter. The spent electrolyte after each process must be disposed of or subjected to regeneration. In addition, without the stage of preliminary thermal depassivation of PGM, the classical electrochlorination method allowed to achieve extraction no higher than $80 \%$.

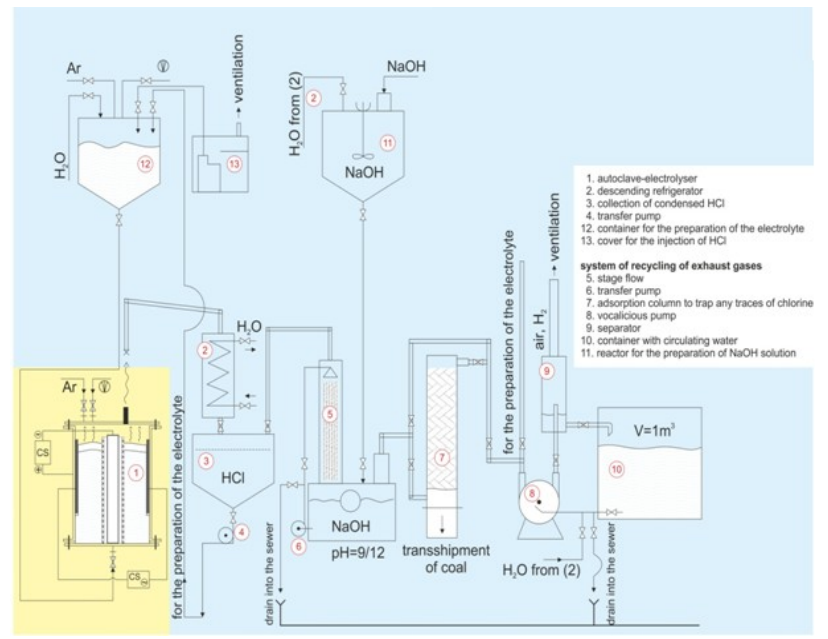

Figure 4. The hardware scheme for third generation installation. Yellow is indicates the part of autoclave solution.

For remedy founded shortages, modernization of electrochemical module for recycling PGM from the spent automobile catalysts based on the described electrochemical method, which allows to achieve high extraction of PGM without stage of thermal activation and provide high-performance and environmental a safe process scheme for its industrial implementation was developed [6]. The scientific approach of the proposed method is the superposition of two independent processes of electrolysis in DC and AC currents under conditions of forced, continuous and complete condensation of electrolyte. Method is protected by two patents $[7,8]$. In the experimental setup of the second generation technical solutions for achievements of high extraction, which are formulated on the basis of a complex of physicochemical investigations, including phase co-standing PGM residuals on the ceramic carrier in the process of electrochlorination. As shown by the research data, the residual metal of PGM on the carrier is present in three main forms. The first is metal on wet ceramics, whose concentration is determined by the content of PGM in the electrolyte, i.e., an open chloride form, is not which has passed into the electrolyte solution. The second is a metal in active form, but not entered into interaction. And the third is a passive unopened form. The typical percentage ratio of the listed forms is the following - $10 \%, 20 \%$, $70 \%$. The residual content of the first two forms is determined by the kinetics electrolytic process and with its optimal is reduced to a minimum. Thus, the residual content of PGM on the carrier is mainly determined by the efficiency of the chlorination and opening of the metal in the active and passive forms.

It is important that the installation involves the imposition of electrochemical processes in a DC and AC currents, and not the use of the result of the classical superposition of an alternating current to a constant one in a single source. This method allowed avoiding a long preliminary stage of thermal activation of PGM. The mechanism of this process is next - the presence of even a small amount of a metal phase (up to $0.2 \%$ mass) in a ceramic raw material under conditions of an external AC current leads to a variable polarization of the active metal centres, turning them into a dipole. At the same time, the entire mass of the bulk material begins to represent a volumetric multipolar electrode, which provides anodic dissolution of the metal in the entire volume of the raw material and subsequent isolation at the cathode upon application of a DC current. Moreover, at a low frequency of the current, the polarity of the dipole alternates with time in time. During the existence of the particle as an anode on it, the electrolyte decomposes and atomic Chlorine is released. Active Chlorine opens the passive form of PGM and dissolves it. However, during the existence of a PGM particle as a cathode, the separation of dissolved PGMs does not have time to occur. In the case of high-frequency alternating current, this effect is absent, and the energy is mainly spent on heating the installation.

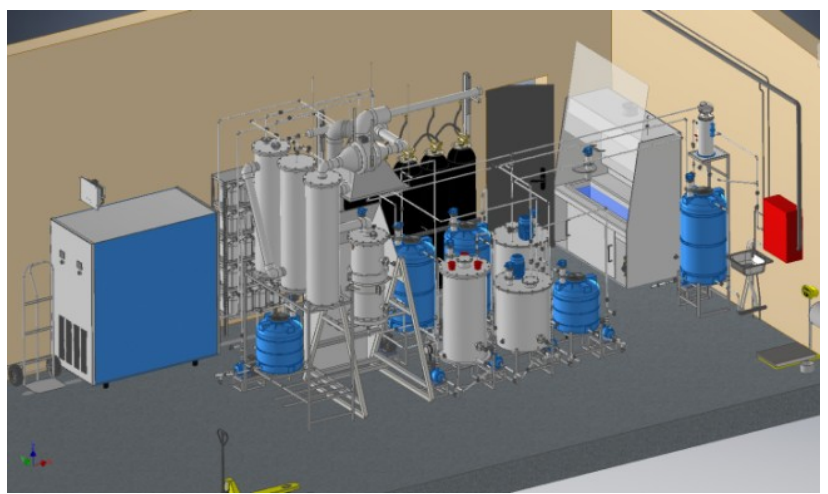

Figure 5. Image of electronic project of third generation installation with closed recycling PGM cycle. Installation has productivity of $50 \mathrm{~kg}$ per working cycle and adapted to location in technical area of 47 sq. meters. 
The DC current from a separate power source (alternative current) provides separation of the dissolved metal on the main cathode and generation on the anode of the atomic Chlorine, which immediately recombines into the molecular one. The latter is the main chlorinating agent already pre-opened with active atomic Chlorine of the bulk of PGM. To effectively remove carrier watersoluble forms of PGM, as well as from electrolyte, platinum electroplating is carried out under conditions constant increase in the concentration of PGM in the electrolyte due to the volume ratio of the solid and liquid phases. This is achieved through forced, continuous evaporation of the electrolyte and its condensation into pure form without impurities in a separate container. This made it possible to increase the precipitation of PGM and to obtain regenerated solutions for their subsequent reuse. Thus, during the process there are absolutely no solutions containing residual PGM and recycling. In addition to the above, this circumstance made it possible to avoid the circulation of the electrolyte, which is difficult to ensure in the conditions of the finely dispersed phase, and to precede the static regime, with an increase in the kinetic parameters of the process. It also made the installation simpler, which is important for industrial implementation.

Under these conditions, the limiting stage of the whole process is to become the evaporation of the electrolyte, the speed of which is easily increased by forced evacuation of its vapours by chemical pumps, and the process of electroprecipitation of PGM in continuously enriched electrolyte occurs rapidly. The complete cycle of processing raw materials under these conditions is 6-8 hours, with direct extraction of PGM Pt$98 \%, \mathrm{Pd}-98 \%$ and $\mathrm{Rh} 90 \%$. Simultaneously with condensation of electrolyte vapours $\left(\mathrm{H}_{2} \mathrm{O}-\mathrm{HCl}\right)$ the capture and neutralization of gaseous products takes place during decomposition $\left(\mathrm{Cl}_{2}\right.$ and $\left.\mathrm{H}_{2}\right)$.

The hardware scheme for installation of third generation is shown in Fig. 4. The The process is carried out with a starting volume ratio of solid and liquid phases which is $1 / 1-1.5$. That makes possible to reduce the amount of technical solutions and is an additional advantage of this method. Now days, work is under way to establish the installation of the third generation, which makes it possible to achieve extraction of rhodium up to $95 \%$ and higher performance (full cycle is 4-5 hours). The main principle of this work is the use of the above factors in the design, and also the ability to conduct the process of electrolysis at elevated temperatures up to 150 ${ }^{0} \mathrm{C}$. The increase in temperature makes it possible additionally and effectively carry out depassivation and chlorination of PGM (mainly Rh) during minimum time. The hardware design (installation design) for the implementation of the technology being developed presupposes an autoclave solution that allows to conduct electrolysis processes at temperatures up to $150{ }^{\circ} \mathrm{C}$ and a pressure up to $5 \mathrm{~atm}$. One of the main technological requirements for design of the system being developed is the reduction of raw material processing in the device, rational (fast and convenient) raw material transfer, explosion safety, remote process control. The installation will have a modular solution for replicating one.

\section{Conclusions}

The proposed technology for recycling catalysts based on the method electrochlorination, has the main advantages:

- High PGM recovery rate in the condition of the direct output with obtaining high grade and easy affined concentrate,

- Minimum number of technological operations and quantity of instrumental devices,

- Stability of obtaining cycling solutions, null waste solutions,

- Ability of using ceramic wastes in the production of heat-insulating materials,

Now the development of a pilot autoclave plant of the third generation with a productivity of $50 \mathrm{~kg}$ per working cycle is completed with its further scaling into an industrial version with a capacity of $400 \mathrm{~kg}$ per working cycle.

Also to increase the efficiency of the recycling process were developed a technology for obtaining moulding high-temperature heat-insulating products by pressing with using ceramic waste processing. Substitution of basic cordierite powder ( ceramic carrier free from PGM) has allowed to reduce the per unit shrinkage of the product by $50-60 \%$, the increase in thermal stability in 1,5-2 times and cost reduction by 15 $20 \%$. In addition, the price of materials for heatinsulating products is several times higher than the price of residual PGM in the cordierite.

\section{Acknowledgement}

This work was supported by the Ministry Education and Science Russian Federation (Grant No. 14.575.21.0153 from 26.09.2017, unique identifier RFMEFI57517X0153).

\section{References}

1. V. Maksimov, Electrochlorination as a method of complex extraction of methal, Moscow: Metallurgy, (1955)

2. M. Kiselev Research laws and technology development extraction of gold by electrochemical chlorination from gold-containing sulfid products, PhD thesis, Ekaterinburg, (2012)

3. K. Konik, E. Kuzas, S. Lobko, S. Naboychenko, Tsvetnyye metally, 12. (2016)

4. A. Antonov and etc., Proc. 7th Int.conf. Russian precious metals and gemstones market. (2004)

5. A. Antonov, Proc. Conf. Committee for Ecology Statetive Duma of the Russian Federation, (2004).

6. A. Antonov and etc., Proc. 30-th ISTC Korea Workshop (2013).

7. A. Antonov, A. Morozov, K. Kryschenko, Russian Patent №198947, (2003)

8. A. Antonov, A. Morozov, A. Novikov, V. Sapylkin, Russian Patent №2540251, (2015) 